\title{
Lupus anticoagulant in patients with COVID-19: A review
}

\author{
Milad Nazari Sabet $^{1}$, Elham Ahmadipour ${ }^{2}{ }^{\circledR}$, Shadi Zamansaraei ${ }^{3^{*}}$ \\ ${ }^{1}$ Department of General Surgery, School of Medicine, Isfahan University of Medical Sciences, Iran \\ ${ }^{2}$ Medical Doctor, Isfahan University of Medical Sciences, Iran \\ ${ }^{3}$ Department of Obstetrics and Gynecology Surgery, Isfahan University of Medical Sciences, Iran
}

Correspondence to:

Shadi Zamansaraei, Email:

shadi.zamansaraei@gmail.com

Received: 22 Apr. 2021

Accepted: 18 June 2021

ePublished: 30 July 2021

Keywords: COVID 19, Lupus anticoagulant, Review article

Citation: Nazari Sabet M, Ahmadipour E, Zamansaraei S. Lupus anticoagulant in patients with COVID-19: a review. J Prev Epidemiol. 2021;6(1):e07. doi: 10.34172/jpe.2021.07

\begin{abstract}
Introduction: Coronavirus disease 2019 (COVID-19) is characterized by a pro-coagulant state that can lead to fatal thromboembolic events. A high prevalence of lupus anticoagulant has been shown in several studies that may at least partially explain the pro-coagulant profile of COVID-19. However, the relation between COVID-19 and lupus anticoagulant is arguable, and no study has clearly evaluated the concussion of lupus anticoagulant on mortality.

Methods: We searched the articles that related to lupus anticoagulant and patients with COVID-19. Two authors independently reviewed the search results to select English language articles discussing lupus anticoagulant in patients with COVID-19.

Results: Recent studies found conflicting results about the association between lupus anticoagulant and thromboembolic complications of COVID-19. Studies documented a high prevalence of lupus anticoagulants as well as several other studies. Patients with lupus anticoagulants were older, and their C-reactive protein, high-sensitivity troponin, and activated partial thromboplastin time (aPTT) were significantly higher than patients without lupus anticoagulants.

Conclusion: Those started on therapeutic anticoagulation showed more severe and complicated involvements and a higher risk of death. According to our results, lupus anticoagulant is highly prevalent among hospitalized COVID-19 patients. Whether these antibodies promote a hypercoagulable state or they are merely a coincidence, epiphenomenon needs further evaluation.
\end{abstract}

\section{A background on lupus anticoagulant \\ What is lupus anticoagulant?}

Lupus anticoagulant refers to a series of various antibodies, which may also be various in behavior among different patients. They are named after their in vitro feature of increasing laboratory coagulation test measures, such as activated partial thromboplastin time (aPTT). It is speculated that these antibodies interfere with the phospholipids that induce in vitro coagulation. In vivo, they probably interact with platelet membrane phospholipids to increase platelet aggregation, which explains their in vivo prothrombotic characteristics. The patient with lupus anticoagulants looks like bleeding on paper but is, in fact, in a thrombotic state (1).

\section{Testing for lupus anticoagulants}

No test alone is conclusively diagnostic for lupus anticoagulants, however multiple tests are needed to tell if the blood sample is positive for lupus anticoagulants. It is also needed to repeat the testing to prove them positive because these antibodies

\section{Key point}

This review shows lupus anticoagulant is highly prevalent among hospitalized COVID-19 patients.

appear due to infection but disappear with the resolution of the infection. The partial thromboplastin time (PTT) represents the clotting time. It can also show that one's blood is positive for anticoagulant antibodies, even though not specifically.

If laboratory tests indicate the presence of anticoagulant antibodies, the tests need to be repeated. Retesting normally happens in about 12 weeks. Lupus anticoagulant binds to phospholipid. If vast amounts of an exogenous phospholipid are added, it can eliminate the lupus anticoagulant in a competitive way.

If PTT indicates the presence of anticoagulant antibodies, further hematologic studies are required to rule out other causes. Although, this is less likely to happen with the dilute Russell viper venom assay $(1,2)$. 


\section{Different types of lupus anticoagulant}

IgG, IgM, and IgA are different isotypes of anticardiolipin antibody. The isotype associated primarily with complications and problems is IgG. The approved test for detecting anticardiolipin antibodies is ELISA (enzymelinked immunosorbent assay) which can detect all of the isotypes at once or independently. Autoimmune hemolytic anemia is a condition associated with high levels of IgM.

ELISA assays are a good option to detect this, since they detect antibodies that bind to beta-2 glycoprotein and/or cardiolipin. Double positive patients for lupus anticoagulant with anti-beta-2 glycoprotein antibodies form clots more probably than those with isolated lupus anticoagulant. Phosphatidylserine is an example of other lupus anticoagulants that bind to other targets $(1,2)$.

What is the clinical significance of lupus anticoagulant? This depends on the clinical context. For example, lupus anticoagulants can rarely be found in asymptomatic people. Transient, infrequent production of lupus anticoagulant may occur by viral infections. These were suggested to increase the risk of thrombosis under some circumstances, but its significance needs yet to be determined. In the late 1940s, an antibody in some lupus patients with an increased clotting test (which depends on phospholipids) was disclosed. Therefore, it was presumed to increase the tendency to bleed, hence the "lupus anticoagulant" which is now considered more as a misnomer, because, on the one hand, the term "anticoagulant" is technically wrong; lupus anticoagulant, in fact, increases the clotting ability of the blood. On the other hand, the term "lupus" is misleading: over half of all people who test positive for this antibody do not have Lupus. This is rather a prothrombotic condition that needs therapeutic anticoagulation (1-3).

\section{Evidence on lupus anticoagulant in SARS and} COVID- 19

Chow et al studied 21 pediatric patients with rather mild SARS. Eight patients (38\%) had an elevated aPTT and tested positive for lupus anticoagulant (4). The prolonged aPTT does not necessarily denote DIC (disseminated intravascular coagulation), and may result from lupus anticoagulant (the same is the case with COVID-19) (4). This study demonstrates that lupus anticoagulant antibodies can occur in relatively mild illnesses due to coronavirus. This might mean that lupus anticoagulant antibody formation occurs earlier in the course of disease pathogenesis (4).

Harzallah et al applied both dilute Russell viper venom test and sensitive aPTT on 56 COVID-19 patients to check them for the presence of lupus anticoagulant. Using IgM and IgG detection, they found that around 25/56 (45\%) were positive for lupus anticoagulant, 5/50 $(10 \%)$ were positive for anticardiolipin antibody or anti- beta-2 glycoprotein antibody, and 6\% tested positive for both. This study lacks some important details (e.g., the titers and exact patterns of anticardiolipin and antibeta-2 glycoprotein antibody) (5).

Beyrouti et al studied six patients with large-vessel occlusive stroke due to COVID-19 in London. These patients appear relatively hypercoagulable (with significant amounts of dimer and ferritin). Five of Six patients had lupus positive anticoagulants without anticardiolipin antibody or glycoprotein-2 antibody. The remaining patients were positive for lupus anticoagulant antibodies and anticardiolipin/anti-beta-2-glycoprotein antibodies. Interestingly, these two-positive patients had the highest D-dimer value (>80000 ug/L) (6).

Interestingly, despite having five lupus anticoagulants in a more accurate trial, only one of these patients had high aPTT levels. This indicates that the standard aPTT level is a sensitive test for lupus anticoagulants. One way to explain this finding is that COVID-19 patients in many cases increase factor VIII, which tends to lower aPTT levels and potentially mask the presence of lupus anticoagulants (6).

To explain the coagulopathy and antiphospholipid antibodies of patients with COVID-19, Zhang et al presented three patients with multiple cerebral infarction and antiphospholipid antibodies. They report that lupus anticoagulants have not been diagnosed, but they do not describe how to evaluate lupus anticoagulants (for example, whether they used two different methods, including a dilute rappel poison test). Interestingly, aPTT was prolonged in all of these patients. I wonder if these patients may actually have lupus anticoagulants that have not been diagnosed by testing (7).

Bowles et al investigated the etiology of prolonged PTT in 216 COVID-19 patients, 44 (20\%) of which had an elevated PTT. Around 35 of the patients with high PTT were further evaluated. Lupus anticoagulant assays were done on 34 patients that 31 of them are positive. The presence of lupus anticoagulant was confirmed using 50:50 mixing studies and the addition of phospholipids. Factor VIII levels were elevated and factor XII decreased. Practically, this pattern of factors should cause hypercoagulation (8).

Low-level of Factor XII is probably irrelevant. Factor XII does not play a very important role in vivo. For example, factor XII deficiency bears few clinical consequences (8).

Factor VIII levels are elevated. This is a more important factor for coagulation, so increasing its level may lead to clinical coagulation. In addition, increased levels of factor VIII may reduce aPTT and thus mask the presence of lupus anticoagulant (8). Unfortunately, these authors did not test specific types of antibodies (for example, anticardiolipin antibodies or 2-glycoprotein antibodies) (8).

In a study described by researchers of American 
Society of Hematology, 27 COVID-19 patients from two institutes were evaluated for lupus anticoagulation. Around 27/20 (74\%) of the patients had a dilute positive Russell horizontal assay, which often occurs significantly. $4.27(15 \%)$ had positive antibody lupus anticoagulant (aPTT-based system). Around 0.27 had IgM or IgG antibodies against cardiolipin or beta-2 glycoprotein. Some salient detail is missing, and other details had a low power $(\mathrm{n}=27)(9)$.

Helms et al in a prospective study described 150 COVID-19 patients in four intensive care units. These patients were compared with a similar number of patients with acute respiratory distress syndrome (ARDS) without COVID-19, and it is unclear whether COVID-19 causes unique coagulation abnormalities (or whether these are just abnormalities that are widespread in serious patients). Patients with COVID-19 had more thrombotic complications than non- COVID-19 patients (e.g., with a pulmonary embolism rate of $12 \%$ versus $2 \%)(10)$. Lupus anticoagulants were evaluated in 57 patients based on clinical suspicion (due to increased PTT or clinical thrombosis). Lupus anticoagulant was found in 57 (around 50 of these patients). The evaluation includes a complete test as described above, including two screening tests (a modified aPTT method and a dilute Russell African toxin) as well as mixing and confirmation studies with the addition of phospholipids (10). Helms et al suggest that lupus anticoagulant may be present in a large proportion of critically ill patients with COVID-19. However, only 57 of 150 patients were evaluated for lupus anticoagulant, and this might be due to selection bias (10).

\section{Synthesis of this data}

By combining the data from these seven sources, we can get an approximate understanding of what is going on here. Lupus anticoagulants are present in many COVID-19 patients if carefully examined after the use of specific tests such as Russell's snake venom test and aPTT (may be approximately 50-75\%, depending on the severity of the disease). Takes. Fewer COVID-19 patients have prolonged aPTT levels using standard methods (perhaps 20). Among these patients, the vast majority (90\%) may have lupus anticoagulants. Most lupus anticoagulants do not have anticardiolipin antibodies or anti-beta-2 glycoprotein antibodies. However, a few patients have a combination of lupus anticoagulant plus anticardiolipin and/or anti-beta-2 glycoprotein antibodies. This small subset of "double positive" patients might mean a predisposition towards thrombosis (e.g., ischemic stroke) (11).

Clinical implications of lupus anticoagulant in COVID - 19

One of the challenges of COVID-19 is the distillation of theoretical information and the emergence of data that is actually relevant to the clinical management of patients. For example, testing all COVID-19 patients for lupus anticoagulants is unlikely to be helpful (because we do not know the clinical implications of this finding).

For the actual management of COVID-19 patients, the following is relevant;

If aPTT is found to be high, this is often a reflection of lupus anticoagulant. Therefore, high aPTT should not be a general contraindication to anticoagulants. If time permits, mixing studies can be performed to confirm the presence of a clotting inhibitor (instead of the clotting factor deficiency, as seen in intravascular clotting). For patients on anticoagulant heparin infusion, it may be preferable to follow the level of anti-Xa activity (e.g., "heparin level") instead of aPTT; particularly, if the baseline aPTT level is abnormal. A major debate in the management of COVID-19 is what anticoagulant dose to use for different patients. It is interesting to guess whether patients with long-term aPTT can represent patients who are prone to clotting due to lupus anticoagulants. However, this requires further research-in particular, to determine whether aPTT can predict thrombosis independently above and beyond the D-dimer level. Lupus anticoagulants are antibodies that prolong coagulation tests in vitro (especially aPTT) but may increase blood clotting in vivo.

Various viral infections (e.g., hepatitis C) can stimulate lupus anticoagulant; this is sometimes correlated with an increase in risk of thrombosis. Specific testing shows that lupus anticoagulant may be present in approximately half of the patients with COVID-19. However, only $\sim 20 \%$ of patients with COVID-19 appear to show high aPTT levels in conventional test methods. If high aPTT levels are seen in a patient with COVID-19, it is more likely to indicate lupus anticoagulant activity (rather than true blood coagulation). Likewise, high aPTT levels should not necessarily be interpreted as contraindications to anticoagulants. If possible, heparin injection may be best titrated for anti-Xa levels instead of aPTT values (because anti-Xa levels may be more reliable and affected by lupus anticoagulants). If present, what role can it play in the pathogenesis of COVID-19? It is conceivable that these antibodies could promote a hypercoagulable state, but it is also possible that they are merely an associative epiphenomenon of the infection (12).

\section{Discussion}

This hypothesis does not explain the lack of prognostic power of lupus anticoagulant. Antiphospholipid autoantibodies (anticardiolipin and anti-beta2glycoprotein) are used with lupus anticoagulant to diagnose antiphospholipid syndrome $(1,3,5)$. Positive anticardiolipin, anti-beta 2-glycoprotein, and anticoagulant lupus in the same subject (triple positivity) is usually associated with a very high thrombotic risk, 
while double positivity (presence of anticardiolipin) and anti-beta 2-glycoprotein, but the absence of lupus anticoagulant is less risky. The risk of thrombosis associated with lupus anticoagulant is unknown. Given that anticardiolipin and antibacterial 2-glycoprotein have not been tested in the present study, we cannot rule out that a small group with triple positivity may have a worse prognosis. However, several studies have found very low levels of antiphospholipid antibody in COVID-19 patients which were unrelated to these antibodies and the prognosis of COVID-19 (13).

Lupus anticoagulant may be a transient event in patients this might explain the lack of association between lupus anticoagulant and a poor prognosis in our study, and therefore, have a limited and temporary pathophysiological effect. Unfortunately, we evaluated lupus anticoagulant when taking it but not during follow-up. This is a study limitation $(2,6,10)$. Another explanation for our finding is that anticoagulation may reduce the thrombotic risk linked to lupus anticoagulant. In fact, all of our patients were initially treated with prophylactic or anticoagulant therapy. In particular, all patients were treated with the prophylactic dose of enoxaparin. Five patients were on therapeutic anticoagulant before admission and it was continued during their hospital stay $(6,10)$.

Patients undergoing preventive coagulation start anticoagulant therapy as soon as a thrombotic complication is suspected or disease progression worsens. The proportion of patients treated with coagulation therapy was significantly higher among non-living subjects than survivors' patients who were started on therapeutic anticoagulation had more severe and complicated presentations of the disease and a higher risk of death. Although the proportion of patients undergoing therapeutic coagulation was higher in patients with lupus coagulation than in patients without lupus coagulation, this difference is not statistically significant. However, we recorded high mortality (14).

This may indicate that the risk of thrombosis remains high due to other mechanisms that do not respond to blood coagulation with heparin, such as acute antithrombin depletion or other autoimmune mechanisms. In addition, there are studies in patients who are very strict and do not have anticoagulant antibodies to lupus and antiphospholipid, but with many coagulation abnormalities that can greatly increase the risk of thrombosis. However, future work should provide more information on a comprehensive and effective assessment of individual thrombotic risk in COVID-19, as recent autopsy findings showed in all patients due to COVID-19 and involvement of the renin-angiotensin system. There is in situ thrombosis. The SARS-CoV-2 virus can enter the host cells by ACE2(angiotensinconverting receptor enzyme 2), and studies have shown that the expression of ACE2 could be associated with
SARS-Cov2 infection (15).

It may be interesting to know how many thrombotic events have occurred in our study. Unfortunately, we cannot provide an exact number because the complications in some patients are not sufficiently recorded, this is a limitation of the study (15).

Interestingly, our study confirms that obesity, low oxygen saturation, and high troponin levels predict mortality and the need for mechanical ventilation, as recently stated. These variables can serve as prognostic markers along with other cardiac and coagulation parameters $(14,15)$.

Overall, our study shows that lupus anticoagulant is common in patients admitted for COVID-19 but does not appear to be a prognostic indicator of mortality and need for mechanical ventilation. The reasons for these findings are still unclear. If the results of our study are confirmed, the anticoagulant drug for lupus in COVID-19 should be considered as a side effect of a higher inflammatory condition or another mechanism than a causative agent of thromboembolism. However, our data confirm the major role of obesity, hypoxia, and elevated troponin levels as predictors of worse prognosis in these patients $(14,15)$.

\section{Authors' contribution}

MNS and SZ prepared the manuscript. EH edited the paper. All authors read and signed the final manuscript.

\section{Conflicts of interest}

The authors declare that they have no competing interests.

\section{Ethical considerations}

Ethical issues (including plagiarism, data fabrication, double publication) have been completely observed by the authors.

\section{Funding/Support}

None.

\section{References}

1. Connell NT, Battinelli EM, Connors JM. Coagulopathy of COVID-19 and antiphospholipid antibodies. J Thromb Haemost. 2020. doi: 10.1111/jth.14893.

2. Schouwers SM, Delanghe JR, Devreese KM. Lupus anticoagulant (LAC) testing in patients with inflammatory status: does C-reactive protein interfere with LAC test results? Thromb Res. 2010;125:102-4. doi: 10.1016/j.thromres.2009.09.001.

3. Abdel-Wahab N, Talathi S, Lopez-Olivo MA, Suarez-Almazor ME. Risk of developing antiphospholipid antibodies following viral infection: a systematic review and meta-analysis. Lupus. 2018;27:572-83. doi: 10.1177/0961203317731532.

4. Chow EY, Chiu WK. Severe acute respiratory syndrome and lupus anticoagulants in children. Br J Haematol. 2003;12:3678. doi: 10.1046/j.1365-2141.2003.04608.x.

5. Harzallah I, Debliquis A, Drénou B. Lupus anticoagulant is frequent in patients with COVID-19. J Thromb Haemost. 2020;18(8):2064-5. doi: 10.1111/jth.14867.

6. Beyrouti R, Adams ME, Benjamin L, Cohen H, Farmer SF, Goh YY, et al. Characteristics of ischaemic stroke associated with COVID-19. J Neurol Neurosurg Psychiatry. 2020;91:889-91. doi: 10.1136/jnnp-2020-323586. 
7. Zhang $\mathrm{Y}$, Xiao $\mathrm{M}$, Zhang $\mathrm{S}$, Xia $\mathrm{P}$, Cao $\mathrm{W}$, Jiang $\mathrm{W}$, et al. Coagulopathy and antiphospholipid antibodies in patients with COVID-19. N Engl J Med. 2020;382:e38. doi: 10.1056/ NEJMc2007575.

8. Bowles L, Platton S, Yartey N, Dave M, Lee K, Hart DP, et al. Lupus anticoagulant and abnormal coagulation tests in patients with COVID-19. N Engl J Med. 2020;383:288-90. doi: 10.1056/NEJMc2013656.

9. American Society of Hematology. COVID-19 and aPL antibodies: frequently asked questions, 2020. Available: https://www.hematology.org/covid-19/covid-19-and-apl-ab. Accessed 23 May 2020

10. Helms J, Tacquard C, Severac F, Leonard-Lorant I, Ohana M, Delabranche $X$, et al. High risk of thrombosis in patients with severe SARS-CoV-2 infection: a multicenter prospective cohort study. Intensive Care Med. 2020;46:1089-98. doi: 10.1007/ s00134-020-06062-x.

11. Pengo V, Tripodi A, Reber G, Rand JH, Ortel TL, Galli M, et al. Update of the guidelines for lupus anticoagulant detection. Subcommittee on Lupus Anticoagulant/Antiphospholipid Antibody of the Scientific and Standardisation Committee of the International Society on Thrombosis and Haemostasis J Thromb Haemost. 2009;7:1737-40. doi: 10.1111/j.15387836.2009.03555.x

12. Wenzel C, Stoiser B, Locker GJ, Laczika K, Quehenberger P, Kapiotis $\mathrm{S}$, et al. Frequent development of lupus anticoagulants in critically ill patients treated under intensive care conditions. Crit Care Med. 2002;30:763-70. doi: 10.1097/00003246200204000-00007.

13. Klok FA, Kruip M, van der Meer NJM, Arbous MS, Gommers $\mathrm{D}$, Kant KM, et al. Incidence of thrombotic complications in critically ill ICU patients with COVID-19. Thromb Res. 2020;191:145-7. doi: 10.1016/j.thromres.2020.04.013.

14. Tang N, Li D, Wang X, Sun Z. Abnormal coagulation parameters are associated with poor prognosis in patients with novel coronavirus pneumonia. J Thromb Haemost. 2020;18:844-7. doi: $10.1111 /$ jth.14768.

15. Tang N, Bai H, Chen X, Gong J, Li D, Sun Z. Anticoagulant treatment is associated with decreased mortality in severe coronavirus disease 2019 patients with coagulopathy. J Thromb Haemost. 2020;18:1094-9. doi: 10.1111/jth.14817. 